\author{
A.T. Assanova ${ }^{1, *}$, Zh.S. Tokmurzin ${ }^{2}$ \\ ${ }^{1}$ Institute of Mathematics and Mathematical Modeling, Almaty, Kazakhstan \\ ${ }^{2}$ K.Zhubanov Aktobe Regional State University, Aktobe, Kazakhstan \\ (E-mail: assanova@math.kz; tokmurzinzh@gmail.com)
}

\title{
Method of functional parametrization for solving a semi-periodic initial problem for fourth-order partial differential equations
}

\begin{abstract}
A semi-periodic initial boundary-value problem for a fourth-order system of partial differential equations is considered. Using the method of functional parametrization, an additional parameter is carried out and the studied problem is reduced to the equivalent semi-periodic problem for a system of integro-differential equations of hyperbolic type second order with functional parameters and integral relations. An interrelation between the semi-periodic problem for the system of integro-differential equations of hyperbolic type and a family of Cauchy problems for a system of ordinary differential equations is established. Algorithms for finding of solutions to an equivalent problem are constructed and their convergence is proved. Sufficient conditions of a unique solvability to the semi-periodic initial boundary value problem for the fourth-order system of partial differential equations are obtained.
\end{abstract}

Keywords: semi-periodic initial boundary-value problem, fourth-order system of partial differential equations, the method of functional parametrization, semi-periodic problem, system of integro-differential equations of hyperbolic type second order, family of Cauchy problems, algorithm, unique solvability.

\section{Introduction}

In the present paper, on the domain $\Omega=[0, T] \times[0, \omega]$ we consider the following semi-periodic initial boundary value problem for a fourth order system of partial differential equations

$$
\begin{gathered}
\frac{\partial^{4} u}{\partial t^{3} \partial x}=A_{1}(t, x) \frac{\partial^{3} u}{\partial t^{2} \partial x}+A_{2}(t, x) \frac{\partial^{3} u}{\partial t^{3}}+A_{3}(t, x) \frac{\partial^{2} u}{\partial t^{2}}+A_{4}(t, x) \frac{\partial^{2} u}{\partial t \partial x}+ \\
+A_{5}(t, x) \frac{\partial u}{\partial t}+A_{6}(t, x) \frac{\partial u}{\partial x}+A_{7}(t, x) u+f(t, x), \\
u(0, x)=\varphi_{1}(x), \quad x \in[0, \omega], \\
\left.\frac{\partial u(t, x)}{\partial t}\right|_{t=0}=\varphi_{2}(x), \quad x \in[0, \omega], \\
\left.\frac{\partial^{2} u(t, x)}{\partial t^{2}}\right|_{t=0}=\left.\frac{\partial^{2} u(t, x)}{\partial t^{2}}\right|_{t=T}, \quad x \in[0, \omega], \\
u(t, 0)=\psi(t), \quad t \in[0, T],
\end{gathered}
$$

where $u(t, x)=\operatorname{col}\left(u_{1}(t, x), \ldots, u_{n}(t, x)\right)$ is unknown function, the $n \times n$ matrices $A_{i}(t, x),(i=\overline{1,7})$, and $n$ vector-function $f(t, x)$ are continuous on $\Omega ; n$ vector-function $\psi(t)$ are continuously three times differentiable on $[0, T]$; the $n$ vector-functions $\varphi_{1}(x)$ and $\varphi_{2}(x)$ are continuously differentiable on $[0, \omega]$.

Let $C\left(\Omega, \mathbb{R}^{n}\right)$ be a space of continuous on $\Omega$ vector functions $u(t, x)$ with the norm

$$
\|u\|_{0}=\max _{(t, x) \in \Omega}\|u(t, x)\|,\|u(t, x)\|=\max _{i=1, n}\left|u_{i}(t, x)\right| .
$$

${ }^{*}$ Corresponding author.

E-mail: assanova@math.kz 
A function $u(t, x) \in C\left(\Omega, \mathbb{R}^{n}\right)$ having partial derivatives

$$
\begin{gathered}
\frac{\partial u(t, x)}{\partial t} \in C\left(\Omega, \mathbb{R}^{n}\right), \frac{\partial u(t, x)}{\partial x} \in C\left(\Omega, \mathbb{R}^{n}\right), \frac{\partial^{2} u(t, x)}{\partial t \partial x} \in C\left(\Omega, \mathbb{R}^{n}\right), \frac{\partial^{2} u(t, x)}{\partial t^{2}} \in C\left(\Omega, \mathbb{R}^{n}\right), \\
\frac{\partial^{3} u(t, x)}{\partial t^{2} \partial x} \in C\left(\Omega, \mathbb{R}^{n}\right), \frac{\partial^{3} u(t, x)}{\partial t^{3}} \in C\left(\Omega, \mathbb{R}^{n}\right), \frac{\partial^{4} u(t, x)}{\partial t^{3} \partial x} \in C\left(\Omega, \mathbb{R}^{n}\right),
\end{gathered}
$$

is called a classical solution to problem (1)-(5) if it satisfies system (1) for all $(t, x) \in \Omega$, and the initial and the boundary conditions (2)-(5).

Mathematical modeling of various processes in physics, ecology, chemistry, biology and others are leaded to initial - boundary value problems for a higher-order partial differential equations with variable coefficients and boundary functions [1,2]. Despite the presence of numerous works, general statements of initial-boundary value problems for the higher-order system of partial differential equations remain poorly studied up to now. Therefore, the problems of solvability of initial-boundary value problems for the fourth-order system of partial differential equations are important in applied problems [1-8]. Some classes of initial-boundary value problems for systems of fourth-order hyperbolic equations are studied in $[4-8]$.

Aim of the paper is to study issues for an existence and uniqueness of classical solutions to the semiperiodic initial boundary value problem for the fourth-order system of partial differential equations (1)-(5). We will establish coefficient criteria for its unique solvability and construct algorithms for finding its approximate solutions. For reaching this goal, we use method of functional parametrization [9-19] for solving the problem (1)-(5).

First, we introduce a new unknown function $w(t, x)=\frac{\partial^{2} u(t, x)}{\partial t^{2}}$ and rewrite problem (1)-(5) in the following from

$$
\begin{gathered}
\frac{\partial^{2} w}{\partial t \partial x}=A_{1}(t, x) \frac{\partial w}{\partial x}+A_{2}(t, x) \frac{\partial w}{\partial t}+A_{3}(t, x) w+f(t, x)+ \\
+A_{4}(t, x) \frac{\partial^{2} u}{\partial t \partial x}+A_{5}(t, x) \frac{\partial u}{\partial t}+A_{6}(t, x) \frac{\partial u}{\partial x}+A_{7}(t, x) u \\
w(0, x)=w(T, x), \quad x \in[0, \omega] \\
w(t, 0)=\ddot{\psi}(t), \quad t \in[0, T], \\
\frac{\partial u}{\partial t}=\varphi_{2}(x)+\int_{0}^{t} w(\tau, x) d \tau, \quad u(t, x)=\varphi_{1}(x)+t \cdot \varphi_{2}(x)+\int_{0}^{t} \int_{0}^{\tau} w\left(\tau_{1}, x\right) d \tau_{1} d \tau \\
\frac{\partial^{2} u}{\partial t \partial x}=\dot{\varphi}_{2}(x)+\int_{0}^{t} \frac{\partial w(\tau, x)}{\partial x} d \tau, \quad \frac{\partial u}{\partial x}=\dot{\varphi}_{1}(x)+t \cdot \dot{\varphi}_{2}(x)+\int_{0}^{t} \int_{0}^{\tau} \frac{\partial w\left(\tau_{1}, x\right)}{\partial x} d \tau_{1} d \tau .
\end{gathered}
$$

A solution of problem (6)-(10) is a function $w(t, x) \in C\left(\Omega, \mathbb{R}^{n}\right)$ having partial derivatives $\frac{\partial w(t, x)}{\partial t} \in$ $\in C\left(\Omega, \mathbb{R}^{n}\right), \frac{\partial w(t, x)}{\partial x} \in C\left(\Omega, \mathbb{R}^{n}\right), \frac{\partial^{2} w(t, x)}{\partial t \partial x} \in C\left(\Omega, \mathbb{R}^{n}\right)$, where the function $u(t, x)$ and its partial derivatives $\frac{\partial u(t, x)}{\partial t}, \frac{\partial u(t, x)}{\partial x}$ and $\frac{\partial^{2} u(t, x)}{\partial t \partial x}$ are determined from integral relations (9), (10).

The method of functional parametrization is based on the introduction of additional parameters as the value of the desired solution on the line $t=0$ of the domain $\Omega$. The semi-periodic boundaryvalue problem for system of hyperbolic equations with integral conditions (6)-(10) is reduced to an equivalent semi-periodic problem for the system of integro-differential equations of hyperbolic type with functional parameter depending on $x$. The properties of solution and its partial derivatives pass into the properties of functional parameter. Using this method, we obtained coefficient conditions for the unique solvability of semi-periodic initial boundary value problem for the fourth-order system of partial differential equations (1)-(5).

Different types of initial-boundary value problems for some classes of fourth-order system of partial differential equations are studied in [20-22] by introducing additional new functions. 
Scheme of the method functional parametrization without partitioning of the domain

We denote by $\lambda(x)=w(0, x)$ and in problem (6) - (10) make the change $\tilde{w}(t, x)=w(t, x)-\lambda(x)$. Then, the integral relations (9) and (10) have the following form

$$
\begin{gathered}
\frac{\partial u(t, x)}{\partial t}=\varphi_{2}(x)+t \cdot \lambda(x)+\int_{0}^{t} \tilde{w}(\tau, x) d \tau, \\
u(t, x)=\varphi_{1}(x)+t \cdot \varphi_{2}(x)+\frac{t^{2}}{2} \cdot \lambda(x)+\int_{0}^{t} \int_{0}^{\tau} \tilde{w}\left(\tau_{1}, x\right) d \tau_{1} d \tau, \\
\frac{\partial^{2} u(t, x)}{\partial t \partial x}=\dot{\varphi}_{2}(x)+t \cdot \dot{\lambda}(x)+\int_{0}^{t} \frac{\partial \tilde{w}(\tau, x)}{\partial x} d \tau, \\
\frac{\partial u(t, x)}{\partial x}=\dot{\varphi}_{1}(x)+t \cdot \dot{\varphi}_{2}(x)+\frac{t^{2}}{2} \cdot \dot{\lambda}(x)+\int_{0}^{t} \int_{0}^{\tau} \frac{\partial \tilde{w}\left(\tau_{1}, x\right)}{\partial x} d \tau_{1} d \tau .
\end{gathered}
$$

Further, in system (6) instead of functions $\frac{\partial u(t, x)}{\partial t}, u(t, x) \frac{\partial^{2} u(t, x)}{\partial t \partial x}$ and $\frac{\partial u(t, x)}{\partial x}$ we substitute their representations from (11)-(14), respectively. We get the following equivalent nonlocal problem for system of integro-differential equations of hyperbolic type with an unknown function $\lambda(x)$ :

$$
\begin{gathered}
\frac{\partial^{2} \tilde{w}}{\partial t \partial x}=A_{1}(t, x) \frac{\partial \tilde{w}}{\partial x}+A_{2}(t, x) \frac{\partial \tilde{w}}{\partial t}+A_{3}(t, x) \tilde{w}+A_{4}(t, x) \int_{0}^{t} \frac{\partial \tilde{w}(\tau, x)}{\partial x} d \tau+ \\
+A_{5}(t, x) \int_{0}^{t} \tilde{w}(\tau, x) d \tau+A_{6}(t, x) \int_{0}^{t} \int_{0}^{\tau} \frac{\partial \tilde{w}\left(\tau_{1}, x\right)}{\partial x} d \tau_{1} d \tau+A_{7}(t, x) \int_{0}^{t} \int_{0}^{\tau} \tilde{w}\left(\tau_{1}, x\right) d \tau_{1} d \tau+ \\
+\left[A_{1}(t, x)+A_{4}(t, x) t+A_{6}(t, x) \frac{t^{2}}{2}\right] \dot{\lambda}(x)+\left[A_{3}(t, x)+A_{5}(t, x) t+A_{7}(t, x) \frac{t^{2}}{2}\right] \lambda(x)+ \\
+f(t, x)+g_{1}(t, x)+g_{2}(t, x), \\
\tilde{w}(0, x)=0, \quad x \in[0, \omega], \\
\tilde{w}(t, 0)=\ddot{\psi}(t)-\ddot{\psi}(0), \quad t \in[0, T], \\
\tilde{w}(T, x)=0, \quad x \in[0, \omega],
\end{gathered}
$$

where $\quad g_{1}(t, x)=A_{4}(t, x) \dot{\varphi}_{2}(x)+A_{5}(t, x) \varphi_{2}(x)$,

$$
g_{2}(t, x)=A_{6}(t, x)\left[\dot{\varphi}_{1}(x)+t \cdot \dot{\varphi}_{2}(x)\right]+A_{7}(t, x)\left[\varphi_{1}(x)+t \cdot \varphi_{2}(x)\right] .
$$

The compatibility condition is valid:

$$
\lambda(0)=\ddot{\psi}(0)
$$

Problems (6)-(10) and (15)-(18) are equivalent in the sense that if the function $w(t, x)$ is a solution of problem (6)-(10), then the pair $\{\lambda(x)=w(0, x), \tilde{w}(t, x)=w(t, x)-w(0, x)\}$ will be a solution of problem (15)-(18), and vice versa, if a pair $\{\lambda(x), \tilde{w}(t, x)\}$ is a solution to problem (15)-(18), then the function $\{\lambda(x)+\tilde{w}(t, x)\}$ will be the solution to problem (6)-(10).

For fixed $\lambda(x), \dot{\lambda}(x)$ the function $\tilde{w}(t, x)$ is a solution to the Goursat problem on $\Omega$ with conditions (16), (17). From (16), (17) we obtain $\frac{\partial \tilde{w}(0, x)}{\partial x}=0, \quad \frac{\partial \tilde{w}(t, 0)}{\partial t}=\dddot{\psi}(t)$ and reduce the Goursat problem to an equivalent system of three integral equations

$$
\begin{gathered}
\frac{\partial \tilde{w}(t, x)}{\partial x}=\int_{0}^{t}\left[A_{1}(\tau, x) \frac{\partial \tilde{w}(\tau, x)}{\partial x}+A_{2}(\tau, x) \frac{\partial \tilde{w}(\tau, x)}{\partial \tau}+A_{3}(\tau, x) \tilde{w}(\tau, x)\right] d \tau+ \\
+\int_{0}^{t}\left[A_{4}(\tau, x) \int_{0}^{\tau} \frac{\partial \tilde{w}\left(\tau_{1}, x\right)}{\partial x} d \tau_{1}+A_{5}(\tau, x) \int_{0}^{\tau} \tilde{w}\left(\tau_{1}, x\right) d \tau_{1}\right] d \tau+
\end{gathered}
$$




$$
\begin{aligned}
& +\int_{0}^{t}\left[A_{6}(\tau, x) \int_{0}^{\tau} \int_{0}^{\tau_{1}} \frac{\partial \tilde{w}\left(\tau_{2}, x\right)}{\partial x} d \tau_{2} d \tau_{1}+A_{7}(\tau, x) \int_{0}^{\tau} \int_{0}^{\tau_{1}} \tilde{w}\left(\tau_{2}, x\right) d \tau_{2} d \tau_{1}\right] d \tau+ \\
& +\int_{0}^{t}\left[A_{1}(\tau, x)+A_{4}(\tau, x) \tau+A_{6}(\tau, x) \frac{\tau^{2}}{2}\right] d \tau \dot{\lambda}(x)+\int_{0}^{t}\left[A_{3}(\tau, x)+A_{5}(\tau, x) \tau+A_{7}(\tau, x) \frac{\tau^{2}}{2}\right] d \tau \lambda(x)+ \\
& +\int_{0}^{t}\left[f(\tau, x)+g_{1}(\tau, x)+g_{2}(\tau, x)\right] d \tau \\
& \frac{\partial \tilde{w}(t, x)}{\partial t}=\dddot{\psi}(t)+\int_{0}^{x}\left[A_{1}(t, \xi) \frac{\partial \tilde{w}(t, \xi)}{\partial \xi}+A_{2}(t, \xi) \frac{\partial \tilde{w}(t, \xi)}{\partial t}+A_{3}(t, \xi) \tilde{w}(t, \xi)\right] d \xi+ \\
& +\int_{0}^{x}\left[A_{4}(t, \xi) \int_{0}^{t} \frac{\partial \tilde{w}(\tau, \xi)}{\partial \xi} d \tau+A_{5}(t, \xi) \int_{0}^{t} \tilde{w}(\tau, \xi) d \tau\right] d \xi+ \\
& +\int_{0}^{x}\left[A_{6}(t, \xi) \int_{0}^{t} \int_{0}^{\tau} \frac{\partial \tilde{w}\left(\tau_{1}, \xi\right)}{\partial \xi} d \tau_{1} d \tau+A_{7}(t, \xi) \int_{0}^{t} \int_{0}^{\tau} \tilde{w}\left(\tau_{1}, \xi\right) d \tau_{1} d \tau\right] d \xi+ \\
& +\int_{0}^{x}\left[A_{1}(t, \xi)+A_{4}(t, \xi) t+A_{6}(t, \xi) \frac{t^{2}}{2}\right] \dot{\lambda}(\xi) d \xi+\int_{0}^{x}\left[A_{3}(t, \xi)+A_{5}(t, \xi) t+A_{7}(t, \xi) \frac{t^{2}}{2}\right] \lambda(\xi) d \xi+ \\
& +\int_{0}^{x}\left[f(t, \xi)+g_{1}(t, \xi)+g_{2}(t, \xi)\right] d \xi \\
& \tilde{w}(t, x)=\dddot{\psi}(t)-\ddot{\psi}(0)+\int_{0}^{x} \frac{\partial \tilde{w}(\tau, \xi)}{\partial \xi} d \xi .
\end{aligned}
$$

Instead of $\frac{\partial \tilde{w}(\tau, x)}{\partial x}, \quad \frac{\partial \tilde{w}\left(\tau_{1}, x\right)}{\partial x}, \quad \frac{\partial \tilde{w}\left(\tau_{2}, x\right)}{\partial x}$ we substitute the corresponding right-hand side of (20) and, repeating this procedure $m(m=1,2,3, \ldots)$ times, we obtain

$$
\frac{\partial \tilde{w}}{\partial x}=D_{m}(t, x) \cdot \dot{\lambda}(x)+E_{m}(t, x) \cdot \lambda(x)+G_{m}\left(t, x, \frac{\partial \tilde{w}}{\partial x}\right)+H_{m}\left(t, x, \frac{\partial \tilde{w}}{\partial t}, \tilde{w}\right)+F_{m}(t, x),
$$

where

$$
\begin{gathered}
D_{m}(t, x)=D_{m}^{(1)}(t, x)+D_{m}^{(2)}(t, x)+D_{m}^{(3)}(t, x), \\
E_{m}(t, x)=E_{m}^{(1)}(t, x)+E_{m}^{(2)}(t, x)+E_{m}^{(3)}(t, x), \\
G_{m}\left(t, x, \frac{\partial \tilde{w}}{\partial x}\right)=G_{m}^{(1)}\left(t, x, \frac{\partial \tilde{w}}{\partial x}\right)+G_{m}^{(2)}\left(t, x, \frac{\partial \tilde{w}}{\partial x}\right)+G_{m}^{(3)}\left(t, x, \frac{\partial \tilde{w}}{\partial x}\right), \\
H_{m}\left(t, x, \frac{\partial \tilde{w}}{\partial t}, \tilde{w}\right)=H_{m}^{(1)}\left(t, x, \frac{\partial \tilde{w}}{\partial t}, \tilde{w}\right)+H_{m}^{(2)}(t, x, \tilde{w})+H_{m}^{(3)}(t, x, \tilde{w}), \\
F_{m}(t, x)=F_{m}^{(1)}(t, x)+F_{m}^{(2)}(t, x)+F_{m}^{(3)}(t, x), \\
D_{m}^{(1)}(t, x)=\int_{0}^{t} A_{1}\left(\tau_{1}, x\right) d \tau_{1}+\int_{0}^{t} A_{1}\left(\tau_{1}, x\right) \int_{0}^{\tau_{1}} A_{1}\left(\tau_{2}, x\right) d \tau_{2} d \tau_{1}+ \\
E_{m}^{(1)}(t, x)=\int_{0}^{t} A_{1}\left(\tau_{1}, x\right) \ldots \int_{0}^{\tau_{m-1}} A_{1}\left(\tau_{m}, x\right) d \tau_{m} \ldots d \tau_{1}, \\
A_{3}\left(\tau_{1}, x\right) d \tau_{1}+\ldots+\int_{0}^{t} A_{1}\left(\tau_{1}, x\right) \ldots \int_{0}^{\tau_{m-2}} A_{1}\left(\tau_{m-1}, x\right) \int_{0}^{\tau_{m-1}} A_{3}\left(\tau_{m}, x\right) d \tau_{m} \ldots d \tau_{1}, \\
G_{m}^{(1)}\left(t, x, \frac{\partial \tilde{w}}{\partial x}\right)=\int_{0}^{t} A_{1}\left(\tau_{1}, x\right) \ldots \int_{0}^{\tau_{m-2}} A_{1}\left(\tau_{m-1}, x\right) \int_{0}^{\tau_{m-1}} A_{1}\left(t_{m}, x\right) \frac{\partial \tilde{w}\left(\tau_{m}, x\right)}{\partial x} d \tau_{m} \ldots d \tau_{1}, \\
H_{m}^{(1)}\left(t, x, \tilde{w}, \frac{\partial \tilde{w}}{\partial t}\right)=\int_{0}^{t}\left[A_{2}\left(\tau_{1}, x\right) \frac{\partial \tilde{w}}{\partial \tau_{1}}+A_{3}\left(\tau_{1}, x\right) \tilde{w}\right] d \tau_{1}+\ldots+
\end{gathered}
$$




$$
\begin{aligned}
& +\int_{0}^{t} A_{1}\left(\tau_{1}, x\right) \ldots \int_{0}^{\tau_{m-2}} A_{1}\left(\tau_{m-1}, x\right) \int_{0}^{\tau_{m-1}}\left[A_{2}\left(\tau_{m}, x\right) \frac{\partial \tilde{w}}{\partial \tau_{m}}+A_{3}\left(\tau_{m}, x\right) \tilde{w}\right] d \tau_{m} \ldots d \tau_{1}, \\
& F_{m}^{(1)}(t, x)=\int_{0}^{t} f\left(\tau_{1}, x\right) d \tau_{1}+\ldots+\int_{0}^{t} A_{1}\left(\tau_{1}, x\right) \ldots \int_{0}^{\tau_{m-2}} A_{1}\left(\tau_{m-1}, x\right) \int_{0}^{\tau_{m-1}} f\left(\tau_{m}, x\right) d \tau_{m} \ldots d \tau_{1} \\
& D_{m}^{(2)}(t, x)=\int_{0}^{t} A_{4}\left(\tau_{1}, x\right) \cdot \tau_{1} d \tau_{1}+\int_{0}^{t} A_{4}\left(\tau_{1}, x\right) \int_{0}^{\tau_{1}} \int_{0}^{\tau_{2}} A_{4}\left(\tau_{3}, x\right) \cdot \tau_{3} d \tau_{3} d \tau_{2} d \tau_{1}+ \\
& +\ldots+\int_{0}^{t} A_{4}\left(\tau_{1}, x\right) \int_{0}^{\tau_{1}} \int_{0}^{\tau_{2}} A_{4}\left(\tau_{3}, x\right) \ldots \int_{0}^{\tau_{2 m-3}} \int_{0}^{\tau_{2 m-2}} A_{4}\left(\tau_{2 m-1}, x\right) \tau_{2 m-1} d \tau_{2 m-1} d \tau_{2 m-2} \ldots d \tau_{1}, \\
& E_{m}^{(2)}(t, x)=\int_{0}^{t} A_{5}\left(\tau_{1}, x\right) \tau_{1} d \tau_{1}+\ldots+ \\
& +\int_{0}^{t} A_{4}\left(\tau_{1}, x\right) \ldots \int_{0}^{\tau_{2 m-5}} \int_{0}^{\tau_{2 m-4}} A_{4}\left(\tau_{2 m-3}, x\right) \int_{0}^{\tau_{2 m-3}} \int_{0}^{\tau_{2 m-2}} A_{5}\left(\tau_{2 m-1}, x\right) \tau_{2 m-1} d \tau_{2 m-1} \ldots d \tau_{1} \\
& G_{m}^{(2)}\left(t, x, \frac{\partial \tilde{w}}{\partial x}\right)=\int_{0}^{t} A_{4}\left(\tau_{1}, x\right) \ldots \int_{0}^{\tau_{2 m-3}} \int_{0}^{\tau_{2 m-2}} A_{4}\left(\tau_{2 m-1}, x\right) \int_{0}^{\tau_{2 m-1}} \frac{\partial \tilde{w}\left(\tau_{2 m}, x\right)}{\partial x} d \tau_{2 m} \ldots d \tau_{1} \\
& H_{m}^{(2)}(t, x, \tilde{w})=\int_{0}^{t} A_{5}\left(\tau_{1}, x\right) \int_{0}^{\tau_{1}} \tilde{w}\left(\tau_{2}, x\right) d \tau_{2} d \tau_{1}+\ldots+\int_{0}^{t} A_{4}\left(\tau_{1}, x\right) \ldots \\
& \ldots \int_{0}^{\tau_{2 m-5}} \int_{0}^{\tau_{2 m-4}} A_{4}\left(\tau_{2 m-3}, x\right) \int_{0}^{\tau_{2 m-3}} \int_{0}^{\tau_{2 m-2}} A_{5}\left(\tau_{2 m-1}, x\right) \int_{0}^{2 m-1} \tilde{w}\left(\tau_{2 m}, x\right) d \tau_{2 m} \ldots d \tau_{1}, \\
& F_{m}^{(2)}(t, x)=\int_{0}^{t} g_{1}\left(\tau_{1}, x\right) d \tau_{1}+\ldots+ \\
& +\int_{0}^{t} A_{4}\left(\tau_{1}, x\right) \ldots \int_{0}^{\tau_{2 m-5}} \int_{0}^{\tau_{2 m-4}} A_{4}\left(\tau_{2 m-3}, x\right) \int_{0}^{\tau_{2 m-3}} \int_{0}^{\tau_{2 m-2}} g_{1}\left(\tau_{2 m-1}, x\right) d \tau_{2 m-1} \ldots d \tau_{1} \\
& D_{m}^{(3)}(t, x)=\int_{0}^{t} A_{6}\left(\tau_{1}, x\right) \cdot \frac{\tau_{1}^{2}}{2} d \tau_{1}+ \\
& +\int_{0}^{t} A_{6}\left(\tau_{1}, x\right) \int_{0}^{\tau_{1}} \int_{0}^{\tau_{2}} \int_{0}^{\tau_{3}} A_{6}\left(\tau_{4}, x\right) \cdot \frac{\tau_{4}^{2}}{2} d \tau_{4} d \tau_{3} d \tau_{2} d \tau_{1}+\ldots+\int_{0}^{t} A_{6}\left(\tau_{1}, x\right) \ldots \\
& \ldots \int_{0}^{\tau_{3 m-8}} \int_{0}^{\tau_{3 m-7}} \int_{0}^{\tau_{3 m-6}} A_{6}\left(\tau_{3 m-5}, x\right) \int_{0}^{\tau_{3 m-5}} \int_{0}^{\tau_{3 m-4}} \int_{0}^{\tau_{3 m-3}} A_{6}\left(\tau_{3 m-2}, x\right) \frac{\tau_{3 m-2}^{2}}{2} d \tau_{3 m-2} \ldots d \tau_{1} \text {, } \\
& E_{m}^{(3)}(t, x)=\int_{0}^{t} A_{7}\left(\tau_{1}, x\right) \frac{\tau_{1}^{2}}{2} d \tau_{1}+\ldots+\int_{0}^{t} A_{6}\left(\tau_{1}, x\right) \ldots \\
& \ldots \int_{0}^{\tau_{3 m-8}} \int_{0}^{\tau_{3 m-7}} \int_{0}^{\tau_{3 m-6}} A_{6}\left(\tau_{3 m-5}, x\right) \int_{0}^{\tau_{3 m-5}} \int_{0}^{\tau_{3 m-4}} \int_{0}^{\tau_{3 m-3}} A_{7}\left(\tau_{3 m-2}, x\right) \frac{\tau_{3 m-2}^{2}}{2} d \tau_{3 m-2} \ldots d \tau_{1}, \\
& G_{m}^{(3)}\left(t, x, \frac{\partial \tilde{w}}{\partial x}\right)= \\
& =\int_{0}^{t} A_{6}\left(\tau_{1}, x\right) \ldots \int_{0}^{\tau_{3 m-5}} \int_{0}^{\tau_{3 m-4}} \int_{0}^{\tau_{3 m-3}} A_{6}\left(\tau_{3 m-2}, x\right) \int_{0}^{\tau_{3 m-2}} \int_{0}^{\tau_{3 m-1}} \frac{\partial \tilde{w}\left(\tau_{3 m}, x\right)}{\partial x} d \tau_{3 m} \ldots d \tau_{1}, \\
& H_{m}^{(3)}(t, x, \tilde{w})=\int_{0}^{t} A_{7}\left(\tau_{1}, x\right) \int_{0}^{\tau_{1}} \int_{0}^{\tau_{2}} \tilde{w}\left(\tau_{3}, x\right) d \tau_{3} d \tau_{2} d \tau_{1}+\ldots+\int_{0}^{t} A_{6}\left(\tau_{1}, x\right) \ldots \int_{0}^{\tau_{3 m-8}} \int_{0}^{\tau_{3 m-7}} \\
& \int_{0}^{\tau_{3 m-6}} A_{6}\left(\tau_{3 m-5}, x\right) \int_{0}^{\tau_{3 m-5}} \int_{0}^{\tau_{3 m-4}} \int_{0}^{\tau_{3 m-3}} A_{7}\left(\tau_{3 m-2}, x\right) \int_{0}^{\tau_{3 m-2}} \int_{0}^{\tau_{3 m-1}} \tilde{w}\left(\tau_{3 m}, x\right) d \tau_{3 m} \ldots d \tau_{1}
\end{aligned}
$$




$$
\begin{gathered}
F_{m}^{(3)}(t, x)=\int_{0}^{t} g_{2}\left(\tau_{1}, x\right) d \tau_{1}+\ldots+ \\
+\int_{0}^{t} A_{6}\left(\tau_{1}, x\right) \ldots \int_{0}^{\tau_{3 m-8}} \int_{0}^{\tau_{3 m-7}} \int_{0}^{\tau_{3 m-6}} A_{6}\left(\tau_{3 m-5}, x\right) \int_{0}^{\tau_{3 m-5}} \int_{0}^{\tau_{3 m-4}} \int_{0}^{\tau_{3 m-3}} g_{2}\left(\tau_{3 m-2}, x\right) d \tau_{3 m-2} \ldots d \tau_{1} .
\end{gathered}
$$

Assumptions regarding the data of problem (6)-(10) allow us to differentiate relation (18) with respect to $x$ :

$$
\frac{\partial \tilde{w}(T, x)}{\partial x}=0 .
$$

Relation (24) will be equivalent to relation (18) if the compatibility condition (19) is satisfied.

From the right-hand side of (23), finding the value of $\tilde{w}(t, x)$ for $t=T$ and substituting it in (24), we obtain a system of $n$ ordinary first-order differential equations that are not resolved with respect to the derivatives:

$$
D_{m}(T, x) \cdot \dot{\lambda}(x)=-E_{m}(T, x) \cdot \lambda(x)-G_{m}\left(T, x, \frac{\partial \tilde{w}}{\partial x}\right)-H_{m}\left(T, x, \frac{\partial \tilde{w}}{\partial t}, \tilde{w}\right)-F_{m}(T, x) .
$$

For fixed $\frac{\partial \tilde{w}}{\partial x}, \frac{\partial \tilde{w}}{\partial t}, \tilde{w}$ system of differential equations (25) with initial condition (19) is the Cauchy problem with respect to $\lambda(x)$ for all $x \in[0, \omega]$. We solve the Cauchy problem (25), (19) using the fundamental matrix.

Let the matrix $D_{m}(T, x)$ be invertible for all $x \in[0, \omega]$ and $\Phi(x)$ the fundamental matrix to system of differential equations

$$
\frac{d \lambda(x)}{d x}=-\left[D_{m}(T, x)\right]^{-1} E_{m}(T, x) \cdot \lambda(x) .
$$

We re-write system $(25)$ in the following form

$$
\dot{\lambda}(x)=-\left[D_{m}(T, x)\right]^{-1} E_{m}(T, x) \cdot \lambda(x)-\tilde{F}\left(T, x, \frac{\partial \tilde{w}}{\partial x}, \frac{\partial \tilde{w}}{\partial t}, \tilde{w}\right),
$$

where

$$
\tilde{F}\left(T, x, \frac{\partial \tilde{w}}{\partial x}, \frac{\partial \tilde{w}}{\partial t}, \tilde{w}\right)=-\left[D_{m}(T, x)\right]^{-1}\left\{G_{m}\left(T, x, \frac{\partial \tilde{w}}{\partial x}\right)+H_{m}\left(T, x, \frac{\partial \tilde{w}}{\partial t}, \tilde{w}\right)+F_{m}(T, x)\right\} .
$$

A solution to the Cauchy problem (27), (19) is written as

$$
\lambda(x)=\Phi(x) \ddot{\psi}(0)+\Phi(x) \int_{0}^{x} \Phi^{-1}(\xi) \tilde{F}\left(T, \xi, \frac{\partial \tilde{w}}{\partial \xi}, \frac{\partial \tilde{w}}{\partial t}, \tilde{w}\right) d \xi, \quad x \in[0, \omega] .
$$

Thus, the invertibility of the matrix $D_{m}(T, x)$ for all $x \in[0, \omega]$ allows us to find a solution to the original problem (1)-(5) by using the fundamental matrix of a system of ordinary differential equations (26) and constructing solutions to the Goursat problem (15)-(17).

Note that a similar technique was applied to the semi-periodic boundary value problem for systems of quasi-linear and semi-linear hyperbolic equations of second-order in [23-24]. These problems were reduced to an equivalent problems, consisting of a family of periodic boundary-value problems for quasilinear and semi-linear ordinary differential equations, respectively, and functional relations. To solve a families of periodic boundary-value problems for ordinary differential the parametrization method were used. Algorithms for finding periodic boundary-value problem's solution for systems of the quasi-linear and semi-linear system of hyperbolic equations are offered. To construct the algorithms were used a solutions to families of Cauchy problems for systems of ordinary differential equations and systems of functional equations with respect to the introduced parameters. This approach allowed to establish sufficient conditions for the existence of an solution to considered problems. 
Algorithm for finding solution to problem (6)-(10)

As well-known, the fundamental matrix can be constructed for a narrow class of differential equations. Therefore, we propose an algorithm for finding an approximate solution to problem (6)-(10) without using the fundamental matrix.

So, the method of functional parametrization divides the process of finding unknown functions into two stages:

1) finding the introduced functional parameter $\lambda(x) \quad(\dot{\lambda}(x))$ from system (25) with condition (19).

2) finding unknown functions $\frac{\partial \tilde{w}(t, x)}{\partial x}, \frac{\partial \tilde{w}(t, x)}{\partial t}, \tilde{w}(t, x)$ from the system of integral equations $(20)-(22)$.

If the functions $\dot{\lambda}(x), \quad \lambda(x)$ are known, then we will find the functions $\frac{\partial \tilde{w}(t, x)}{\partial x}, \frac{\partial \tilde{w}(t, x)}{\partial t}, \tilde{w}(t, x)$ to solve the system of integral equations (20)-(22), and the function $\lambda(x)+\tilde{w}(t, x)$ will be the solution to problem (6)-(10). If the functions $\frac{\partial \tilde{w}(t, x)}{\partial x}, \frac{\partial \tilde{w}(t, x)}{\partial t}, \tilde{w}(t, x)$ are known, then solving system of differential equations (25) with condition (19), we find $\dot{\lambda}(x), \quad \lambda(x)$ and again determining the sum of the functions $\lambda(x)+\tilde{w}(t, x)$ we find a solution to problem $(6)-(10)$.

Here unknown are both the functions $\dot{\lambda}(x), \lambda(x)$ and the functions $\frac{\partial \tilde{w}(t, x)}{\partial x}, \frac{\partial \tilde{w}(t, x)}{\partial t}, \tilde{w}(t, x)$. Therefore, we use an iterative method and the solution to system of integral equations (20)-(22) and the Cauchy problem $(25),(19)$ is found as the limits of the sequences $\left\{\dot{\lambda}(x), \lambda(x), \frac{\partial \tilde{w}(t, x)}{\partial x}, \frac{\partial \tilde{w}(t, x)}{\partial t}, \tilde{w}(t, x)\right\}$, determined by the following algorithm:

Step 0. Assuming on the right-hand side of $(25) \quad \lambda(x)=\ddot{\psi}(0), \frac{\partial \tilde{w}(t, x)}{\partial x}=0, \frac{\partial \tilde{w}(t, x)}{\partial t}=\dddot{\psi}(t)$, $\tilde{w}(t, x)=\dddot{\psi}(t)-\ddot{\psi}(0)$, and taking into account the invertibility of the matrix $D_{m}(T, x)$ for all $x \in[0, \omega]$, we find $\dot{\lambda}^{(0)}(x)$ from equation (25). Using conditions (19) we find the function $\lambda^{(0)}(x): \lambda^{(0)}(x)=\ddot{\psi}(0)+\int_{0}^{x} \dot{\lambda}^{(0)}(\xi) d \xi, \quad x \in[0, \omega]$. From the system of integral equations (20)-(22), where $\lambda(x)=\lambda^{(0)}(x), \dot{\lambda}(x)=\dot{\lambda}^{(0)}(x)$, we define the functions $\frac{\partial \tilde{w}^{(0)}(t, x)}{\partial x}, \frac{\partial \tilde{w}^{(0)}(t, x)}{\partial t}, \tilde{w}^{(0)}(t, x)$ for all $(t, x) \in \Omega$.

Step 1. From equation (25), where on the right-hand side of $\lambda(x)=\lambda^{(0)}(x), \frac{\partial \tilde{w}(t, x)}{\partial x}=\frac{\partial \tilde{w}^{(0)}(t, x)}{\partial x}$, $\frac{\partial \tilde{w}(t, x)}{\partial t}=\frac{\partial \tilde{w}^{(0)}(t, x)}{\partial t}, \tilde{w}(t, x)=\tilde{w}^{(0)}(t, x)$, by virtue of the invertibility of $D_{m}(T, x)$ for all $x \in[0, \omega]$, we find $\dot{\lambda}^{(1)}(x)$.

Using conditions (19) again, we find the function $\lambda^{(1)}(x)=\ddot{\psi}(0)+\int_{0}^{x} \dot{\lambda}^{(1)}(\xi) d \xi, \quad x \in[0, \omega]$. From the system of integral equations (20)-(22), where $\lambda(x)=\lambda^{(1)}(x), \dot{\lambda}(x)=\dot{\lambda}^{(1)}(x)$, we define the functions $\frac{\partial \tilde{w}^{(1)}(t, x)}{\partial x}, \frac{\partial \tilde{w}^{(1)}(t, x)}{\partial t}, \tilde{w}^{(1)}(t, x)$ for all $(t, x) \in \Omega$.

And so on.

Step $k$. From equation (25), where on the right-hand side of $\lambda(x)=\lambda^{(k-1)}(x), \frac{\partial \tilde{w}(t, x)}{\partial x}=\frac{\partial \tilde{w}^{(k-1)}(t, x)}{\partial x}$, $\frac{\partial \tilde{w}(t, x)}{\partial t}=\frac{\partial \tilde{w}^{(k-1)}(t, x)}{\partial t}, \tilde{w}(t, x)=\tilde{w}^{(k-1)}(t, x)$, by virtue of the reversibility of $D_{m}(T, x)$ for all $x \in[0, \omega]$ we find $\dot{\lambda}^{(k)}(x)$.

Using conditions (19), we find the function $\lambda^{(k)}(x)=\ddot{\psi}(0)+\int_{0}^{x} \dot{\lambda}^{(k)}(\xi) d \xi, \quad x \in[0, \omega]$. From the system of integral equations (20)-(22), where $\lambda(x)=\lambda^{(k)}(x), \dot{\lambda}(x)=\dot{\lambda}^{(k)}(x)$, we define the functions $\frac{\partial \tilde{w}^{(k)}(t, x)}{\partial x}, \frac{\partial \tilde{w}^{(k)}(t, x)}{\partial t}, \tilde{w}^{(k)}(t, x)$ for all $(t, x) \in \Omega$.

Here $k=1,2,3, \ldots$.

The following statement gives conditions for the convergence of the proposed algorithm and the unique solvability of problem (6)-(10) in terms of the initial data.

Theorem 1. Suppose that for some $m, m=1,2,3, \ldots$, the $n \times n$-matrix $D_{m}(T, x)$ is invertible for all $x \in[0, \omega]$ and the following inequalities hold:

a) $\left\|\left[D_{m}(T, x)\right]^{-1}\right\| \leq \gamma_{m}(T, x)$, and $\gamma_{m}(T, x)$ is a positive continuous function for all $x \in[0, \omega]$; 
b) $q_{m}(T, x)=\gamma_{m}(T, x) \cdot\left\{e^{\alpha(x) T}-1-\alpha(x) T-\ldots-\frac{1}{m !}[\alpha(x) T]^{m}\right\} \leq \chi<1$, where $\alpha(x)=\max _{t \in[0, T]}\left(\left\|A_{1}(t, x)\right\|,\left\|A_{4}(t, x)\right\|,\left\|A_{6}(t, x)\right\|\right), \chi$ is constant.

Then there is a unique solution $w^{*}(t, x)$ to problem (6)-(10), determining by equality

$$
w^{*}(t, x)=\lambda^{*}(x)+\tilde{w}^{*}(t, x)
$$

with

$$
\begin{gathered}
\frac{\partial u^{*}(t, x)}{\partial t}=\varphi_{2}(x)+t \cdot \lambda^{*}(x)+\int_{0}^{t} \tilde{w}^{*}(\tau, x) d \tau, \\
u^{*}(t, x)=\varphi_{1}(x)+t \cdot \varphi_{2}(x)+\frac{t^{2}}{2} \cdot \lambda^{*}(x)+\int_{0}^{t} \int_{0}^{\tau} \tilde{w}^{*}\left(\tau_{1}, x\right) d \tau_{1} d \tau, \\
\frac{\partial^{2} u^{*}(t, x)}{\partial t \partial x}=\dot{\varphi}_{2}(x)+t \cdot \dot{\lambda}^{*}(x)+\int_{0}^{t} \frac{\partial \tilde{w}^{*}(\tau, x)}{\partial x} d \tau, \\
\frac{\partial u^{*}(t, x)}{\partial x}=\dot{\varphi}_{1}(x)+t \cdot \dot{\varphi}_{2}(x)+\frac{t^{2}}{2} \cdot \dot{\lambda}^{*}(x)+\int_{0}^{t} \int_{0}^{\tau} \frac{\partial \tilde{w}^{*}\left(\tau_{1}, x\right)}{\partial x} d \tau_{1} d \tau,
\end{gathered}
$$

where $\quad \lambda^{*}(x)=\lim _{k \rightarrow \infty} \lambda^{(k)}(x), \quad \dot{\lambda}^{*}(x)=\lim _{k \rightarrow \infty} \dot{\lambda}^{(k)}(x)$ for all $\quad x \in[0, \omega]$,

$$
\tilde{w}^{*}(t, x)=\lim _{k \rightarrow \infty} \tilde{w}^{(k)}(t, x), \quad \frac{\partial \tilde{w}^{*}(t, x)}{\partial x}=\lim _{k \rightarrow \infty} \frac{\partial \tilde{w}^{(k)}(\tau, x)}{\partial x} \text { for all }(t, x) \in \Omega
$$

Proof of the Theorem 1 is provided according to proposed algorithm above.

Therefore, from the equivalence of problems (6)-(10) and (1)-(5) it follows

Theorem 2. Suppose that for some $m, m=1,2,3, \ldots$, the $n \times n$-matrix $D_{m}(T, x)$ is invertible for all $x \in[0, \omega]$ and the inequalities a), b) of Theorem 1 are fulfilled.

Then there is a unique classical solution $u^{*}(t, x)$ to problem (1)-(5), defining from the following integral representation

$$
u^{*}(t, x)=\varphi_{1}(x)+t \cdot \varphi_{2}(x)+\int_{0}^{t} \int_{0}^{\tau} w^{*}\left(\tau_{1}, x\right) d \tau_{1} d \tau, \quad(t, x) \in \Omega .
$$

\section{Acknowledgments}

This research was funded by the Science Committee of the Ministry of Education and Science of the Republic of Kazakhstan (Grant No. AP08955461).

\section{References}

1 Пташник Б.И. Некорректные граничные задачи для дифференциальных уравнений с частными производными / Б.И. Пташник. - Киев: Наук. думка, 1984. - 264 с.

2 Нахушев А.М. Задачи со смещением для уравнений в частных производных / A.M. Нахушев. - М.: Наука, 2006. - 287 с.

3 Kiguradze I. On solvability of boundary value problems for higher order nonlinear hyperbolic equations [Electronic resource] / I. Kiguradze, T. Kiguradze // Nonlinear Analysis. - 2008. Vol. 69. - No. 7. - P. 1914-1933. - Access mode: https://doi.org/10.1016/j.na.2007.07.033

4 Kiguradze T. On the Dirichlet problem for fourth order linear hyperbolic equations [Electronic resource] / T. Kiguradze, V. Lakshmikantham // Nonlinear Analysis. - 2002. - Vol. 49. P. 197-219. - Access mode: https://doi.org/ 10.1016/S0362-546X(01)00101-8.

5 Midodashvili B. A nonlocal problem for fourth order hyperbolic equations with multiple characteristics / B. Midodashvili // Electr. J. of Differ. Equ. - 2002. - Vol. 2002. - No. 85. P. 1-7. 
6 Midodashvili B. Generalized Goursat problem for a spatial fourth order hyperbolic equation with dominated low terms / B. Midodashvili // Proc. of A. Razmadze Math. Institute. - 2005. - Vol. 138. - P. 43-54.

7 Kiguradze T. On solvability and well-posedness of boundary value problems for nonlinear hyperbolic equations of the fourth order / T. Kiguradze // Georgian Math. J. - 2008. - Vol. 15. P. 555-569.

8 Ferraioli D.C. Fourth order evolution equations which describe pseudospherical surfaces [Electronic resource] / D.C. Ferraioli, K. Tenenblat // J. Differ. Equ. - 2014. - Vol. 257. - P. 3165-3199. - Access mode: https://doi. org/10.1016/j.jde.2014.06.010.

9 Asanova A.T. Well-posedness of nonlocal boundary value problems with integral condition for the system of hyperbolic equations [Electronic resource] / A.T. Asanova, D.S. Dzhumabaev // J. Math. Anal. Appl. - 2013. - Vol. 402. - No. 1. - P. 167-178. - Access mode: https://doi.org /10.1016/j.jmaa.2013.01.012.

10 Asanova A.T. On solvability of nonlinear boundary value problems with integral condition for the system of hyperbolic equations [Electronic resource] / A.T. Asanova // Electronic J. Qualitative Theory of Differ. Equ. - 2015. - Vol. 2015. - No. 63. - P. 1-13. - Access mode: https://doi.org /10.14232/ejqtde.2015.1.63

11 Assanova A.T. Nonlocal problem with integral conditions for systems of hyperbolic equations in characteristic rectangle [Electronic resource] / A.T. Assanova // Russian Mathematics (Iz.VUZ). - 2017. - Vol. 61. - No. 5. - P. 7-20. - Access mode: https://doi.org/10.3103/S10663 69X17050 024

12 Assanova A.T. Solvability of a nonlocal problem for a hyperbolic equation with integral conditions / A.T. Assanova // Electronic J. Differ. Equ. - 2017. - Vol. 2017. - No. 170. - P. 1-12.

13 Assanova A.T. Periodic solutions in the plane of system of second-order hyperbolic equations / A.T. Assanova // Math. Notes. - 2017. - Vol. 101. - No. 1. - P. 39-47. https://doi.org/10.1134 /S0001434617010047

14 Assanova A.T. On a nonlocal problem with integral conditions for the system of hyperbolic equations [Electronic resource] / A.T. Assanova // Differ. Equ. - 2018. - Vol. 54. - No. 2. P. 201-214. - Access mode: https://doi.org /10.1134/S0012266118020076

15 Assanova A. An integral-boundary value problem for a partial differential equation of second order [Electronic resource] / A. Assanova // Turkish J. Math. - 2019. - Vol. 43. - No. 4. P. 1967-1978. - Access mode: https://doi.org /10.3906/mat-1903-111

16 Asanova A.T. One approach to the solution of a nonlocal problem for systems of hyperbolic equations with integral conditions [Electronic resource] / A.T. Assanova // J. Math. Sciences (United States). - 2019. - Vol. 238. - No. 3. - P. 189-206. - Access mode: https://doi.org /10.1007/s10958-019-04228-7

17 Assanova A.T. Solution of initial-boundary value problem for a system of partial differential equations of the third order / A.T. Assanova // Russian Mathematics (Iz.VUZ). - 2019. Vol. 63. - No. 4. - P. 12-22. https://doi.org/10.3103/S1066369X19040029

18 Assanova A.T. On the solvability of a nonlocal problem for the system of Sobolev-type differential equations with integral condition / A.T. Assanova // Georgian Math. J. Published Online: 02/19/2019. https:// doi.org/10.1515/ gmj-2019-2011.

19 Assanova A.T. Solvability of nonlocal problems for systems of Sobolev-type differential equations with a multipoint condition / A.T. Assanova, A.E. Imanchiyev, Z.M. Kadirbayeva // Russian Mathematics. - 2019. - Vol. 63. - No. 12. - P. 1-13. https://doi.org/10.3103/S1066369X1 9120016 
20 Assanova A.T. On the initial-boundary value problem for system of the partial differential equations of fourth order / A.T. Assanova, A.A. Boichuk, Zh.S. Tokmurzin // News of the NAS RK. Physico-Mathem. Ser. - 2019. - Vol. 323. - No. 1. - P. 14-21. https://doi.org/10.32014/2019.2518-1726.2.

21 Assanova A.T. On two-point initial boundary value problem for fourth order partial differential equations / A.T. Assanova, Zh.S. Tokmurzin // Kazakh Mathematical Journal. - 2019. - Vol. 19. - No. 3. - P. 66-78.

22 Assanova A.T. A nonlocal problem for loaded partial differential equations of fourth order A.T. Assanova, A.E. Imanchiyev, Z.M. Kadirbayeva // Bulletin of the Karaganda university Series Mathematics. - 2020. - Vol. 97. - No. 1. - P. 6-16. https://doi.org/10.31489/2020M1/616

23 Orumbayeva N.T. On an algorithm of finding periodical boundary value problem for system of the quasi-linear of hyperbolic equations / N.T. Orumbayeva // Siberian Electronic Mathematical Reports. - 2013. - No. 10. - P. 464-474.

24 Orumbaeva N.T. On solvability of non-linear semi-periodic boundary-value problem for system of hyperbolic equations / N.T. Orumbaeva // Russian Mathematics (Iz.VUZ). - 2016. - Vol. 60. - No. 9. - P. 23-37. https://doi.org/ 10.3103/S1066369X16090036

25 Dzhumabayev D.S. Criteria for the unique solvability of a linear boundary-value problem for an ordinary differential equation / D.S. Dzhumabayev // U.S.S.R. Comput. Math. and Math. Phys. - 1989. - Vol. 29. - No. 1. - P. 34-46.

\title{
А.Т. Асанова, Ж.С. Токмурзин
}

\section{Төртінші ретті дербес туындылы дифференциалдық теңдеулер үшін жартылайпериодты бастапқы есепті шешудің функционалдық параметрлеу әдісі}

\begin{abstract}
Төртінші ретті дербес туындылы дифференциалдық теңдеулер жүйесі үшін жартылайпериодты бастапқы шеттік есеп қарастырылды. Функционалдық параметрлеу әдісі көмегімен авторлар қосымша параметрін енгізіп, зерттеліп отырған есеп екінші ретті гиперболалық тектес интегралдық-дифференциалдық теңдеулер жүйесі үшін функционалдық параметрлері мен интегралдық қатынастары бар пара-пар жартылайпериодты есепке келтірді. Гиперболалық тектес интегралдық-дифференциалдық теңдеулер жүйесі үшін жартылайпериодты есеп пен жай дифференциалдық теңдеулер жүйесі үшін Коши есептері әулетінің өзара байланысы тағайындалған. Пара-пар есептің шешімін табу алгоритмдері құрылған және олардың жинақтылығы дәлелденген. Төртінші ретті дербес туындылы дифференциалдық теңдеулер үшін жартылайпериодты бастапқы шеттік есептің бірмәнді шешілімділігінің жеткілікті шарттары алынған.
\end{abstract}

Kiлm сөздер: жартылайпериодты бастапқы шеттік есеп, төртінші ретті дербес туындылы дифференциалдық теңдеулер жүйесі, функционалдық параметрлеу әдісі, жартылайпериодты есеп, екінші ретті гиперболалық тектес интегралдық-дифференциалдық теңдеулер жүйесі, Коши есептерінің әулеті, алгоритм, бірмәнді шешілімділік. 


\title{
Метод функциональной параметризации решения полупериодической начальной задачи для дифференциальных уравнений в частных производных четвертого порядка
}

\begin{abstract}
Рассмотрена полупериодическая начальная краевая задача для системы дифференциальных уравнений в частных производных четвертого порядка. Авторами с помощью метода функциональной параметризации введен дополнительный параметр, и исследуемая задача сведена к эквивалентной полупериодической задаче для системы интегро-дифференциальных уравнений гиперболического типа второго порядка с функциональными параметрами и интегральными соотношениями. Установлена взаимосвязь полупериодической задачи для системы интегро-дифференциальных уравнений гиперболического типа и семейства задач Коши для системы обыкновенных дифференциальных уравнений. Построены алгоритмы нахождения решений эквивалентной задачи и доказана их сходимость. Получены достаточные условия однозначной разрешимости полупериодической начальной краевой задачи для системы дифференциальных уравнений в частных производных четвертого порядка.
\end{abstract}

Ключевые слова: полупериодическая начальная краевая задача, система дифференциальных уравнений в частных производных четвертого порядка, метод функциональной параметризации, полупериодическая задача, система интегро-дифференциальных уравнений гиперболического типа второго порядка, семейство задач Коши, алгоритм, однозначная разрешимость.

\section{References}

1 Ptashnyck, B.I. (1984). Nekorrektnye hranichnye zadachi dlia differentsialnykh uravnenii s chastnymi proizvodnymi [Ill-Posed Boundary-Value Problems for Partial Differential Equations]. Kiev: Naukova dumka [in Russian].

2 Nakhushev, A.M. (2006). Zadachi so smesheniem dlia uravnenii v chastnykh proizvodnykh /Problems with shift for partial differential equations]. Moscow: Nauka [in Russian].

3 Kiguradze, I., \& Kiguradze, T. (2008). On solvability of boundary value problems for higher order nonlinear hyperbolic equations. Nonlinear Analysis, Vol. 69, No. 7, 1914-1933.

4 Kiguradze, T., \& Lakshmikantham, V. (2002). On the Dirichlet problem for fourth order linear hyperbolic equations. Nonlinear Analysis, Vol. 49, No. 2, 197-219.

5 Midodashvili, B. (2002). A nonlocal problem for fourth order hyperbolic equations with multiple characteristics. Electr. J. of Differ. Equ., Vol. 2002, No. 85, 1-7.

6 Midodashvili, B. (2005). Generalized Goursat problem for a spatial fourth order hyperbolic equation with dominated low terms. Proc. of A. Razmadze Math. Institute, Vol. 138, 43-54.

7 Kiguradze, T. (2008). On solvability and well-posedness of boundary value problems for nonlinear hyperbolic equations of the fourth order. Georgian Math. J., Vol. 15, No. 3, 555-569.

8 Ferraioli, D.C., \& Tenenblat, K. (2014). Fourth order evolution equations which describe pseudospherical surfaces. J. Differ. Equ., Vol. 157, 3165-3199.

9 Asanova, A.T., \& Dzhumabaev, D.S. (2013). Well-posedness of nonlocal boundary value problems with integral condition for the system of hyperbolic equations. J. Math. Anal. Appl., Vol. 402, No. $1,167-178$.

10 Asanova, A.T. (2015). On solvability of nonlinear boundary value problems with integral condition for the system of hyperbolic equations. Electronic J. Qualitative Theory of Differ. Equ., Vol. 63, $1-13$.

11 Assanova, A.T. (2017). Nonlocal problem with integral conditions for systems of hyperbolic equations in characteristic rectangle. Russian Mathematics (Iz.VUZ). Vol. 61, No. 5, 7-20. 
12 Assanova, A.T. (2017). Solvability of a nonlocal problem for a hyperbolic equation with integral conditions. Electronic J. Differ. Equ., Vol. 2017, No. 170, 1-12.

13 Assanova, A.T. (2017). Periodic solutions in the plane of system of second-order hyperbolic equations. Math. Notes., Vol. 101, No. 1, 39-47.

14 Assanova, A.T. (2018). On a nonlocal problem with integral conditions for the system of hyperbolic equations. Differ. Equ., Vol. 54, No. 2, 201-214.

15 Assanova, A. (2019). An integral-boundary value problem for a partial differential equation of second order. Turkish J. Math., Vol. 43, No. 4, 1967-1978.

16 Asanova, A.T. (2019). One approach to the solution of a nonlocal problem for systems of hyperbolic equations with integral conditions. J. Math. Sciences (United States)., Vol. 238, No. 3, 189-206.

17 Assanova, A.T. (2019). Solution of initial-boundary value problem for a system of partial differential equations of the third order. Russian Mathematics (Iz.VUZ), Vol. 63, No. 4, 12-22.

18 Assanova, A.T. (2019). On the solvability of a nonlocal problem for the system of Sobolev-type differential equations with integral condition. Georgian Math. J. Published Online: 02/19/2019; DOI: https:// doi.org/10.1515/ gmj-2019-2011.

19 Assanova, A.T., Imanchiyev, A.E., \& Kadirbayeva, Z.M. (2019). Solvability of nonlocal problems for systems of Sobolev-type differential equations with a multipoint condition. Russian Mathematics (Iz.VUZ), Vol. 63, No. 12, 1-13.

20 Assanova, A.T., Boichuk, A.A., \& Tokmurzin, Zh.S. (2019). On the initial-boundary value problem for system of the partial differential equations of fourth order. News of the NAS RK. PhysicoMathem. Ser., Vol. 323, No. 1, 14-21.

21 Assanova, A.T., \& Tokmurzin, Zh.S. (2019). On two-point initial boundary value problem for fourth order partial differential equations. Kazakh Mathematical Journal, Vol. 19, No. 3, 66-78.

22 Assanova, A.T., Imanchiyev, A.E., \& Kadirbayeva, Z.M. (2020). A nonlocal problem for loaded partial differential equations of fourth order. Bulletin of the Karaganda university - Series Mathematics, Vol. 97, No. 1, 6-16.

23 Orumbayeva, N.T. (2013). On an algorithm of finding periodical boundary value problem for system of the quasi-linear of hyperbolic equations. Siberian Electronic Mathematical Reports, Vol. 10, 464-474.

24 Orumbaeva, N.T. (2016). On solvability of non-linear semi-periodic boundary-value problem for system of hyperbolic equations. Russian Mathematics (Iz.VUZ), Vol. 60, No. 9, 23-37.

25 Dzhumabayev, D.S. (1989). Criteria for the unique solvability of a linear boundary-value problem for an ordinary differential equation. U.S.S.R. Comput. Math. and Math. Phys., Vol. 29, No. 1, $34-46$. 